\title{
Stellung und Aufgaben der Deutschen Akkreditierungsstelle (DAkkS)
}

\author{
Andreas Steinhorst \\ Deutsche Akkreditierungsstelle GmbH (DAkkS), Berlin, Deutschland
}

Die Deutsche Akkreditierungsstelle GmbH (DAkkS) wurde Anfang 2010 als nationale Akkreditierungsstelle der Bundesrepublik Deutschland eingerichtet. Ihr gesetzlicher Auftrag ist die Akkreditierung von Konformitätsbewertungsstellen (wie z.B. Laboratorien) oder Zertifizierungsstellen. Sie ist zugleich die einzige Stelle in Deutschland, die mit dieser Aufgabe betraut ist. Die DAkkS hat ihren Hauptsitz in Berlin und ist an 2 weiteren Standorten vertreten.

In rund 4500 Verfahren begutachtet und überwacht die DAkkS als unabhängige Einrichtung die fachliche Kompetenz von akkreditierten Laboratorien sowie Inspektions- und Zertifizierungsstellen, deren Dienstleistungen in allen Bereichen der Wirtschaft - oder etwa des Gesundheitssektors - benötigt werden. Mit einer Akkreditierung bestätigt die DAkkS, dass diese Stellen ihre Aufgaben nach geltenden Anforderungen erfüllen. Sie leistet so einen wichtigen Beitrag zur Stärkung der Qualitätsinfrastruktur und damit des Wirtschaftsstandortes Deutschland.

Die rechtliche Grundlage für die Arbeit der DAkkS ist die europäische Verordnung (EG) 765/2008 [1] zur «Akkreditierung und Marktüberwachung im Zusammenhang mit der Vermarktung von Produkten». Darin sind den Mitgliedstaaten klare und einheitliche Vorgaben für die Organisation und Durchführung von Akkreditierungen gegeben. Die Verordnung umfasst im Kern folgende Aspekte:

- Mitgliedstaaten hatten zum 1. Januar 2010 jeweils eine einzige nationale Akkreditierungsstelle einzurichten,

- Akkreditierung ist eine Tätigkeit im öffentlichen Interesse und muss hoheitlich wahrgenommen werden,

- die Akkreditierungsstelle muss unabhängig von den zu begutachtenden Konformitätsbewertungsstellen sein,

- Gewährleistung von Kompetenz, Objektivität und Unparteilichkeit sowie die Sicherstellung der Vertraulichkeit,

- keine Gewinnorientierung,
- kein Wettbewerb mit anderen nationalen Akkreditierungsstellen, grenzüberschreitende Akkreditierung stark reglementiert,

- keine eigene Durchführung von Konformitätsbewertungen, keine kommerziellen Beratungsdienstleistungen.

Der deutsche Gesetzgeber hat die Vorgaben der Verordnung (EG) Nr. 765/2008 mit der Verabschiedung des Akkreditierungsstellengesetzes (AkkStelleG) [2] vom 31. Juli 2009 umgesetzt. Mit der Gründung der DAkkS wurde das bis dato zersplitterte deutsche Akkreditierungssystem gebündelt und durch eine einzige Akkreditierungsstelle ersetzt. Damit wird ein Wettbewerb auf Ebene der Akkreditierung vermieden; dies steht bewusst im Gegensatz zum durchaus erwünschten - Wettbewerb auf Ebene der Zertifizierungen. Auch sind nationale Akkreditierungsstellen im Rahmen ihrer hoheitlichen Tätigkeit in der Regel nicht grenzüberschreitend tätig. Somit können sich in Deutschland ansässige Antragsteller ausschließlich über die DAkkS akkreditieren lassen. Weitere Belange sind in den zugehörigen Verordnungen (Beleihungsverordnung [3], Kostenverordnung [4], Akkreditierungssymbolverordnung [5]) geregelt. Die DAkkS ist als $\mathrm{GmbH}$ organisiert und wird im Bereich Gesundheit durch das Bundesministerium für Gesundheit beaufsichtigt.

Bestehende Akkreditierungen der in der DAkkS aufgegangenen Akkreditierungsstellen (Deutsche Gesellschaft für Akkreditierung GmbH (DGA), Deutsche Akkreditierungsstelle Chemie $\mathrm{GmbH}(\mathrm{DACH})$, Deutsches Akkreditierungssystem Prüfwesen (DAP), TGA GmbH/DATech und Deutscher Kalibrierdienst (DKD)) bzw. weiterer behördlicher und privater Akkreditierungsstellen behalten bis zu ihrem Auslaufen (spätestens zum 31. Dezember 2014) ihre Gültigkeit. Sofern diese in der Datenbank der akkreditierten Stellen gelistet sind, werden sie fortan durch die DAkkS überwacht.

\section{KARGER}

Fax +497614520714

Information@Karger.de

www.karger.com 


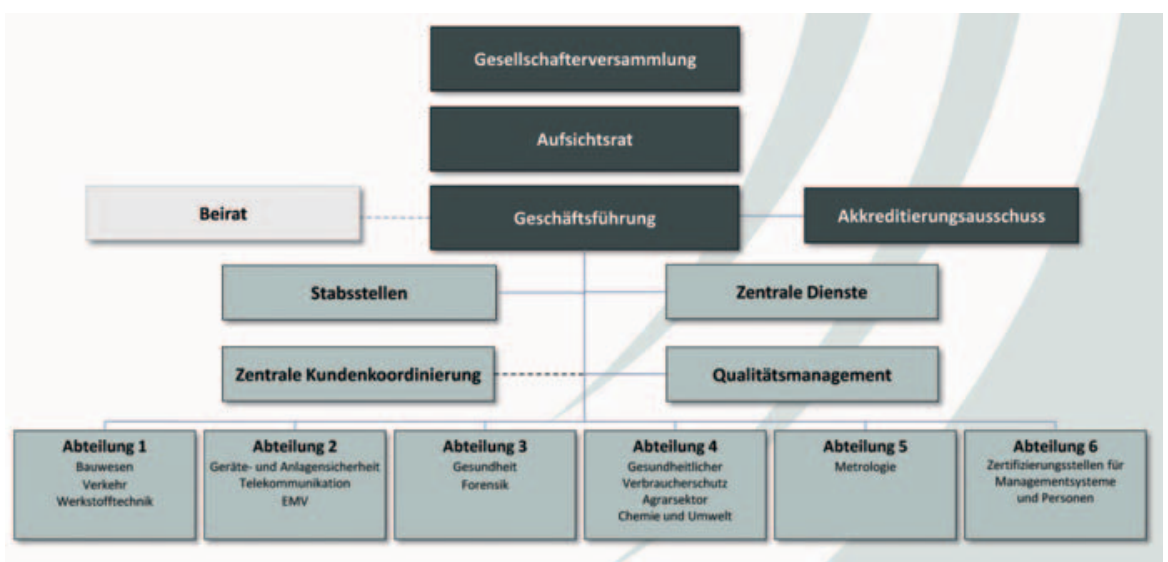

Abb. 1. Organisationsplan der DAkkS.

\begin{tabular}{|l|}
\hline \multicolumn{1}{|c|}{ Abteilung 3} \\
Gesundheit | Forensik \\
\hline Fachbereiche: \\
- Aktive implantierbare medizinische \\
Geräte \\
- Arbeitsmedizin \\
- Arzneimittel und Wirkstoffe, \\
Apotheken \\
- Forensische Medizin, Toxikologie, \\
Biologie \\
- Gendiagnostik \\
- Gesundheitsversorgung \\
- Hygiene \\
- In-vitro-Diagnostika \\
- Kriminaltechnik \\
- Laboratoriumsdiagnostik \\
- Medizinprodukte \\
- Patientennahe Untersuchungen \\
(POCT) \\
- Pflege \\
- Reproduktionsmedizin \\
- Veterinärmedizin \\
\hline
\end{tabular}

\begin{tabular}{|l|}
\multicolumn{1}{|c|}{ Abteilung 6} \\
\multicolumn{1}{|c|}{ Zertifizierungsstellen für } \\
Managementsysteme und \\
Personen
\end{tabular}

Abb. 2. Fachbereiche der Abteilungen 3 und 6 der DAkkS.

Die Geschäftsführung ist die oberste Leitung der DAkkS. Sie führt die Geschäfte nach den Bestimmungen der einschlägigen Rechtsvorschriften und technischen Normen, des Gesellschaftsvertrages, ihrer Geschäftsführerverträge und nach den Gesellschafterbeschlüssen. Zudem vertritt die Geschäftsführung die Interessen der Gesellschaft nach außen.

Neben den Stabsstellen und den Zentralen Diensten sind 6 Fachabteilungen mit insgesamt 94 zugeordneten Fachbereichen für das Akkreditierungsgeschäft der DAkkS zuständig (Abb. 1), wobei für medizinische bzw. gesundheitliche Belange insbesondere die Fachabteilung 3 (Gesundheit/Forensik) mit Sitz in Frankfurt am Main zuständig ist. Dort befindet sich auch die Abteilung 6 der DAkkS, die sich ausschließlich mit der Akkreditierung von Zertifizierungsstellen für Managementsysteme und Personen befasst (Abb. 2).

Um die Aufgaben als unabhängige Akkreditierungsstelle wahrnehmen zu können, hat die DAkkS mehrere Gremien eingerichtet. Dazu gehört unter anderem ein Beirat, der sich aus den Mitgliedern des Akkreditierungsbeirats des Bundesministeriums für Wirtschaft und Technologie (BMWi) zusam- mensetzt. Zentrale Funktion der Beiräte ist die Beratung und Unterstützung der Bundesregierung und der Deutschen Akkreditierungsstelle in Fragen der Akkreditierung. Darüber hinaus unterstützen externe Fachleute in derzeit 28 Sektorkomitees die DAkkS in den zugeordneten Fachbereichen im Hinblick auf die technischen Anforderungen zur Akkreditierung und auf technische Angelegenheiten des Betreibens des Akkreditierungssystems. Dazu gehört auch das Sektorkomitee «Medizinische Laboratorien», in dem auch die Deutsche Gesellschaft für Hämatologie und Onkologie (DGHO) vertreten ist.

\section{DAkkS prüft die Prüfer}

Die DAkkS selbst führt keine Zertifizierungen durch, sondern bewertet die Kompetenz der Zertifizierungsstellen 


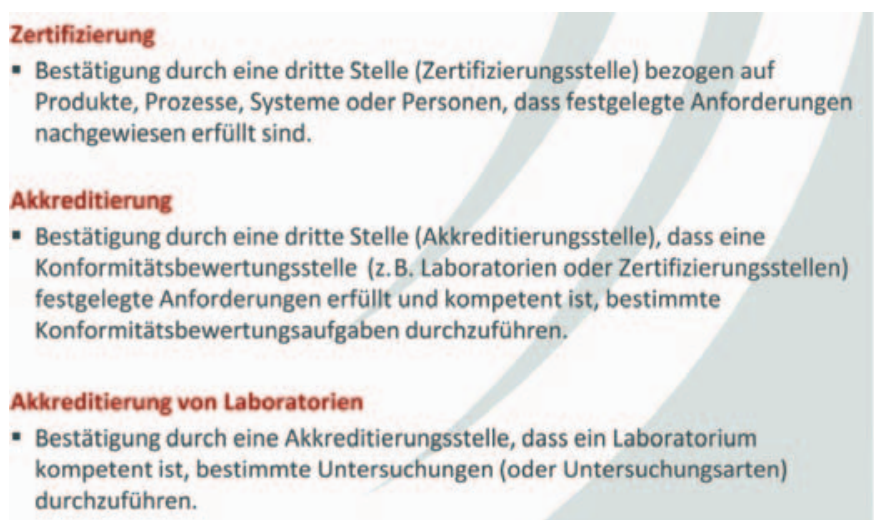

Abb. 3. Akkreditierung versus Zertifizierung.

(Abb. 3). Da Laboratorien in Analogie zu den Zertifizierungsstellen ebenfalls als Konformitätsbewertungsstellen einzuordnen sind, übernimmt die DAkkS auch die Akkreditierung von Laboratorien.

Die Akkreditierungsaktivitäten der DAkkS decken ein großes Spektrum ab (Abb. 4). Die internationale Harmonisierung der dabei zugrunde liegenden Normen gewährleistet, dass die Akkreditierung weltweit nach gleichen Voraussetzungen erfolgt. Durch diese harmonisierten Normen und dank internationaler Abkommen werden die Bewertungsleistungen der in Deutschland akkreditierten Stellen in vielen anderen Ländern Europas und der Welt anerkannt. Damit folgt das internationale Akkreditierungssystem dem Prinzip: einmal geprüft, überall akzeptiert.

Bei den Anforderungen für eine Akkreditierung bindet die DAkkS die jeweiligen Fachkreise in ihre Arbeit ein. So ist die DGHO als Fachgesellschaft bereits seit vielen Jahren an der Akkreditierung von medizinischen Laboratorien durch Mitarbeit an den Checklisten für klinische Chemie und Hämatologie beteiligt. Zertifizierer von Managementsystemen - etwa der Zertifizierung von onkologischen Zentren arbeiten nach der Norm DIN EN ISO/IEC 17021, deren Einhaltung im Zuge der Akkreditierung von der DAkkS überprüft wird. Entsprechende Akkreditierungssysteme sind im Aufbau.

\section{Ablauf der Akkreditierung}

Ein Akkreditierungsverfahren wird auf Antrag eröffnet. Es folgen Antragsprüfung, Vorbegehung (optional) und Sichtung der erforderlichen Dokumente. Nach der Begutachtung vor Ort wird dem Antragsteller und dem Akkreditierungsausschuss ein Begutachtungsbericht überstellt. Bei positiver Begutachtung und Entscheidung durch den Akkreditierungsausschuss wird ein Akkreditierungsbescheid in Verbindung mit einer Akkreditierungsurkunde ausgestellt und es erfolgt die Aufnahme in das Verzeichnis der akkreditierten Stellen. Die
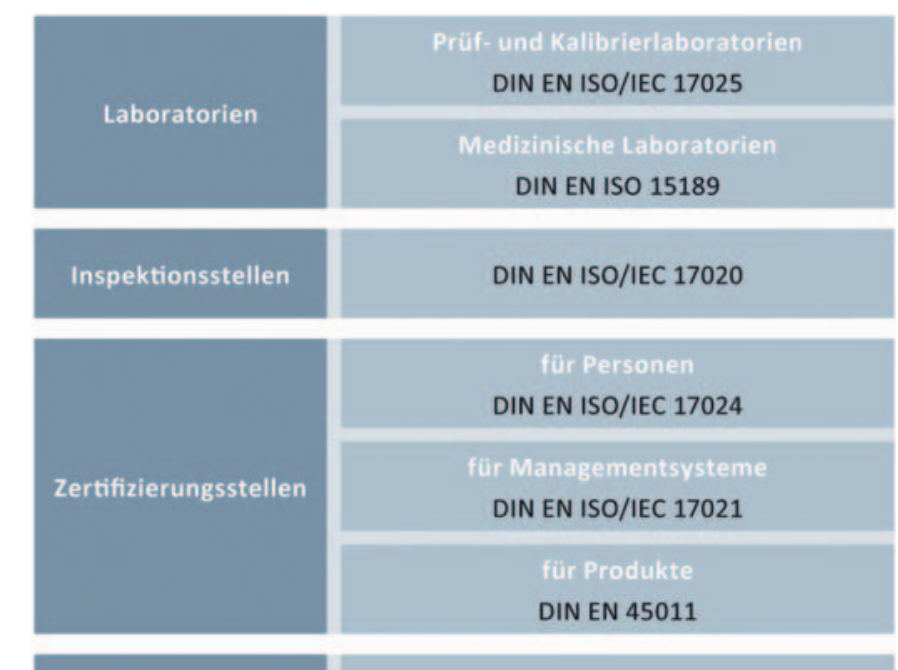

Ringversuchsanbieter

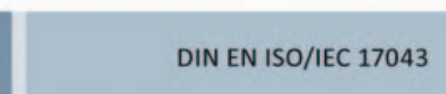

Referenzmaterialhersteller

DIN EN ISO/IEC 17025 mit ISO Guide 34

Abb. 4. Akkreditierungsaktivitäten der DAkkS.

Akkreditierung wird in regelmäßigen Abständen überwacht und erfordert eine Re-Akkreditierung nach 5 Jahren, um ihre Gültigkeit zu behalten.

\section{Welche Vorteile bieten Akkreditierungen?}

- Für Unternehmen: Akkreditierungen erleichtern Unternehmen die Auswahl eines kompetenten Dienstleisters für die verlässliche Bewertung ihrer Waren und Dienstleistungen. Sie fördern die internationale Vergleichbarkeit und Anerkennung von Zertifikaten oder Prüfberichten. Diese Transparenz erleichtert Unternehmen den Zugang zu nationalen und internationalen Märkten - oder ermöglicht ihn erst.

- Für Kunden/Verbraucher/Patienten: Akkreditierungen erhöhen das Vertrauen der Verbraucher in die Qualität und Sicherheit von Produkten oder Dienstleistungen.

- Für akkreditierte Stellen: Akkreditierungen sind ein objektiver Beleg für die Güte der Bewertungstätigkeit. Sie weisen die Kompetenz einer Konformitätsbewertungsstelle nach internationalen Standards aus und schaffen Wettbewerbsvorteile gegenüber nicht akkreditierten Marktteilnehmern.

\section{Wie erkennt man eine akkreditierte Stelle?}

Alle durch die DAkkS akkreditierten Stellen können ihren Status durch eine Akkreditierungsurkunde nachweisen. Im hoheitlichen Bereich ist die Urkunde mit dem Bundesadler 
versehen. Auf Prüfberichten oder Zertifikaten dürfen die Stellen ihren akkreditierten Status zudem durch die Verwendung des DAkkS-Akkreditierungssymbols signalisieren und so die hohe Qualität ihrer Bewertungsleistungen dokumentieren. Das Symbol besteht aus dem geschützten Zeichen der DAkkS und einer eindeutigen Registrierungsnummer.

Bis zum Ablauf aller bis Ende 2009 erteilten Akkreditierungen (also spätestens bis Ende 2014) sind auch die Urkunden und Symbole der ehemaligen Akkreditierungsstellen noch gültig, sofern diese Verfahren durch die DAkkS überwacht werden.

Eine Datenbank aller akkreditierten Stellen stellt die DAkkS auf ihrer Website (www.dakks.de) bereit.

\section{Disclosure Statement}

Es bestehen keine Interessenkonflikte.

\section{Literatur}

1 Verordnung (EG) Nr. 765/2008 des Europäischen Parlaments und des Rates vom 9. Juli 2008 über die Vorschriften für die Akkreditierung und Marktüberwachung im Zusammenhang mit der Vermarktung von Produkten und zur Aufhebung der Verordnung (EWG) Nr. 339/93 des Rates (Amtsblatt der Europäischen Union, L 218, 13. August 2008, S. 82ff.)
2 Akkreditierungsstellengesetz (AkkStelleG) vom 31. Juli 2009 (BGBl. I S. 2625)

3 Verordnung über die Beleihung der Akkreditierungsstelle nach dem Akkreditierungsstellengesetz (AkkStelleGBV) vom 21. Dezember 2009 (BGBl. I S. 3962).
4 Kostenverordnung der Akkreditierungsstelle (AkkStelleKostV) vom 21. Dezember 2009 (BGBl. I S. 3964).

5 Verordnung zur Gestaltung und Verwendung des Akkreditierungssymbols der Akkreditierungsstelle (SymbolVO) vom 15. Dezember 2009 (BGBl. I S. 3870). 